\title{
Nonlinear effects of micro-cracks on acoustic surface and wedge waves
}

\author{
M. Rjelka ${ }^{1}$, P.D. Pupyrev ${ }^{2,3}$, B. Koehler ${ }^{1}$, and A.P. Mayer $^{3}$ \\ ${ }^{I}$ Fraunhofer IKTS, D-01109 Dresden, Germany \\ ${ }^{2}$ Prokhorov General Physics Institute of the Russian Academy of Sciences, Moscow 119991, Russia \\ ${ }^{3}$ HS Offenburg, University of Applied Sciences, D-77723 Gengenbach, Germany \\ E-mail: andreas.mayer@hs-offenburg.de
}

Received March 12, 2018, published online May 28, 2018

\begin{abstract}
Micro-cracks give rise to non-analytic behavior of the stress-strain relation. For the case of a homogeneous spatial distribution of aligned flat micro-cracks, the influence of this property of the stress-strain relation on harmonic generation is analyzed for Rayleigh waves and for acoustic wedge waves with the help of a simple micromechanical model adopted from the literature. For the efficiencies of harmonic generation of these guided waves, explicit expressions are derived in terms of the corresponding linear wave fields. The initial growth rates of the second harmonic, i.e., the acoustic nonlinearity parameter, has been evaluated numerically for steel as matrix material. The growth rate of the second harmonic of Rayleigh waves has also been determined for microcrack distributions with random orientation, using a model expression for the strain energy in terms of strain invariants known in a geophysical context.
\end{abstract}

PACS: $43.35 .+\mathrm{d}$ Ultrasonics, quantum acoustics, and physical effects of sound;

46.40.Cd Mechanical wave propagation (including diffraction, scattering, and dispersion);

62.30. $+\mathrm{d}$ Mechanical and elastic waves; vibrations.

Keywords: micro-cracks, surface acoustic waves, wedge waves, nonlinearity, harmonic generation.

\section{Introduction}

Rayleigh waves and wedge waves are two different types of guided acoustic waves. The strain field associated with the prior (more generally: surface acoustic waves, SAWs) is localized at the surface of a solid, i.e., a twodimensional manifold, whereas the latter have strains localized at a one-dimensional manifold, namely the apex line of a solid elastic wedge, i.e., the intersection line of two surfaces. Straight-crested time-harmonic SAWs are characterized by a two-dimensional wave-vector, whereas the wave-vector of a time-harmonic wedge wave is always along the apex line and hence one-dimensional. Rayleigh waves are studied since the pioneering work by Lord Rayleigh [1] and find technical applications in various fields of science and engineering. Acoustic wedge waves were discovered much later [2,3] and are yet awaiting practical use in technical devices, while prototypes for such devices have already been developed (see, for example, [4,5] for recent reviews).
Both types of guided acoustic waves have in common the property of being non-dispersive in the ideal case. This means that their phase velocity is independent of their frequency. Ideal means essentially a homogeneous elastic medium, planar surfaces and a perfectly sharp wedge tip. The absence of dispersion favors nonlinear effects, as it guarantees phase matching for the growth of higher harmonics.

In crystal lattices, the elastic nonlinearity stems from the interatomic forces in the neighborhood of the rest positions of the atoms, which allows the potential energy of the solid to be expanded in powers of the Green-Lagrange strain tensor [6] and consequently, stress is an analytic function of strain. The influence of the third-order terms in this expansion on the propagation of surface and, to a lesser extent of wedge acoustic waves, has been investigated in theory and experiment (see the recent review [4], and [7]).

Defects like dislocations or micro-cracks are known to strongly modify the nonlinear properties of acoustic waves in solids, while they often change their linear properties 
like their speed to a much lesser degree [8,9]. In the case of bulk and surface acoustic waves, this fact is used for purposes of non-destructive evaluation $[10,11]$ as it provides a viable tool for pre-fatigue detection, even if the size of the micro-cracks is much smaller than the wavelength of the acoustic waves. A central quantity in this context is the acoustic nonlinearity parameter (ANP), which is a measure of the efficiency of second harmonic generation of a timeharmonic fundamental wave. In the case of acoustic wedge waves, no experimental work is known to us that would focus on the influence of dislocations or micro-cracks of sub-wavelength size on the nonlinear propagation properties. However, recent experiments on wedge waves in a polycrystalline aluminum sample containing residual stresses point to unusual, "non-classical" nonlinear behavior [12].

Micro-cracks can render the stress-strain relation of the solid non-analytic. Kinks in this relation are known to be generated by flat micro-cracks of the "kissing bond" type [9] due to the difference of their elastic response to tensile and compressive stress. Non-analytic behavior of the stress-strain relation has also been found in finite element simulations of solids with micro-cracks that have an internal structure [13].

Our goal in this contribution is to present a method of calculating the growth of higher harmonics and of the products for a time-harmonic Rayleigh or wedge wave in an elastic medium with a homogeneous spatial distribution of flat micro-cracks with sub-wavelength size. Concerning their orientation, we consider the simple case of all microcracks being aligned such that their surface normals are all along the same direction. In the case of Rayleigh waves, we choose this direction to be the propagation direction. In the case of wedge waves propagating at a rectangular edge, the alignment is chosen such that the surface normals of the micro-cracks are vertical to the apex line and parallel to one of the surfaces of the wedge. Based on an effective stress-strain relation for this system that follows from a simple micro-mechanical model [14-17] and is supported by finite element simulations, an asymptotic expansion of the displacement field is derived that yields a set of coupled evolution equations for the slowly varying amplitudes of the fundamental and higher harmonics. This approach differs from an earlier study by Oberhardt et al. who used the finite element method to simulate the nonlinear propagation of surface acoustic waves in a medium containing micro-cracks [18].

If the matrix material of the elastic medium with microcracks is isotropic, a totally random distribution of microcrack orientations maintains the isotropy. An expression for the density of potential energy set up by Lyakhovsky and Myasnikov [19] applies to this situation and contains a term that is not analytic in the strain invariants. We shall apply this expression as a model for the elastic properties of media with random micro-crack orientations. Our contribution concludes with a short summary and discussion.

\section{Stress-strain relation for an elastic medium with micro-cracks}

The following derivation will be confined to flat microcracks that are homogeneously distributed in an isotropic matrix material. In the first part of this section, we consider the case of all micro-cracks having the same orientation such that their surface normals are oriented along the $x_{1}$ direction. Micro-mechanical models of penny-shaped micro-cracks in the context of nonlinear acoustic wave effects [20,14-17] make use of an additive decomposition of the total macroscopic infinitesimal strain parameters $\varepsilon_{\alpha \beta}$ into the infinitesimal strain $\varepsilon_{\alpha \beta}^{(0)}$ generated by a macroscopic Cauchy stress $\sigma_{\alpha \beta}$ in the absence of micro-cracks and a separate contribution $\varepsilon_{\alpha \beta}^{(M C)}$ of the micro-cracks. Here and in the following, Cartesian indices are denoted by small Greek letters, and we invoke the convention that summation over repeated Cartesian indices is implied. Applying the approach in [15] to a distribution of fully aligned micro-cracks in the static limit, we obtain

$$
\begin{aligned}
& \varepsilon_{\alpha \beta}^{(M C)}=s_{N} H\left(\sigma_{11}\right) \delta_{\alpha 1} \delta_{\beta 1} \sigma_{11}+ \\
& +s_{S}\left(1+\frac{\sigma_{11} \bar{\mu}}{\sqrt{\sigma_{12}^{2}+\sigma_{13}^{2}}} H\left(-\sigma_{11}\right)\right) \times H\left(1+\frac{\sigma_{11} \bar{\mu}}{\sqrt{\sigma_{12}^{2}+\sigma_{13}^{2}}} H\left(-\sigma_{11}\right)\right) \times \\
& \times\left[\left(\delta_{\alpha 2} \delta_{\beta 1}+\delta_{\alpha 1} \delta_{\beta 2}\right) \sigma_{21}+\left(\delta_{\alpha 3} \delta_{\beta 1}+\delta_{\alpha 1} \delta_{\beta 3}\right) \sigma_{31}\right] .
\end{aligned}
$$

In (1), $H$ denotes the Heaviside step function, $s_{N}, s_{S}$ are coefficients which are proportional to the concentration of the penny-shaped micro-cracks and depend on their diameter and the elastic properties of the matrix material. The quantity $\bar{\mu}$ is the coefficient of friction between the adjacent faces of a micro-crack.

Inserting (1) in the stress-strain relation for the matrix material without micro-cracks,

$$
\sigma_{\alpha \beta}=C_{\alpha \beta \mu \nu}^{(0)} \varepsilon_{\mu \nu}^{(0)}=C_{\alpha \beta \mu \nu}^{(0)}\left(\varepsilon_{\mu \nu}-\varepsilon_{\mu \nu}^{(\mathrm{MC})}\right)
$$

where $C_{\alpha \beta \mu \nu}^{(0)}$ are the elastic constants of the matrix material, we obtain for the diagonal elements of the Cauchy stress and infinitesimal strain tensor:

$$
\begin{aligned}
& \sigma_{11}=c_{11}^{(0)} \varepsilon_{11}+c_{12}^{(0)}\left(\varepsilon_{22}+\varepsilon_{33}\right)+\Delta \sigma_{11}, \\
& \sigma_{22}=c_{11}^{(0)} \varepsilon_{22}+c_{12}^{(0)}\left(\varepsilon_{11}+\varepsilon_{33}\right)+\Delta \sigma_{22}, \\
& \sigma_{33}=c_{11}^{(0)} \varepsilon_{33}+c_{12}^{(0)}\left(\varepsilon_{11}+\varepsilon_{22}\right)+\Delta \sigma_{33},
\end{aligned}
$$

where the stress deviations

$$
\begin{gathered}
\Delta \sigma_{11}=\tau_{0} \varepsilon_{0}+\tau_{1}\left|\varepsilon_{0}\right|, \\
\Delta \sigma_{22}=\Delta \sigma_{33}=\frac{c_{12}^{(0)}}{c_{11}^{(0)}} \Delta \sigma_{11}
\end{gathered}
$$


result from the micro-cracks, and where we have defined the quantity

$$
\varepsilon_{0}=\varepsilon_{11}+\frac{c_{12}^{(0)}}{c_{11}^{(0)}}\left(\varepsilon_{22}+\varepsilon_{33}\right)
$$

$c_{11}^{(0)}, c_{12}^{(0)}$ denote two independent elastic constants of the matrix material in Voigt notation.

The constants $\tau_{0}, \tau_{1}$ in (4) are related to the coefficients $s_{N}, s_{S}$ in (1) via

$$
\begin{gathered}
\tau_{0}=\frac{1}{2} c_{11}^{(0)} \frac{2+c_{11}^{(0)} s_{N}}{1+c_{11}^{(0)} s_{N}}, \\
\tau_{1}=-\frac{1}{2} c_{11}^{(0)} \frac{c_{11}^{(0)} s_{N}}{1+c_{11}^{(0)} s_{N}} .
\end{gathered}
$$

The occurrence of the modulus in (4) introduces nonlinearity into this stress-strain relation.

In the case of shear stress / strain, we consider here only two limiting cases concerning the friction between the faces of the micro-cracks. When the friction is negligible $(\bar{\mu} \rightarrow 0)$, we obtain from (1)

$$
\sigma_{12}=2 c_{66} \varepsilon_{12}, \sigma_{13}=2 c_{66} \varepsilon_{13}, \sigma_{23}=2 c_{44} \varepsilon_{23},
$$

where $c_{44}=c_{44}^{(0)}$ and

$$
c_{66}=\frac{c_{44}^{(0)}}{1+2 c_{44}^{(0)} s_{S}}
$$

In this case, the relation between shear stress and shear strain remains linear, but causes the macroscopic elastic properties of the elastic medium to become anisotropic.

In the case of very large friction $(\bar{\mu} \rightarrow \infty)$, (9) is replaced by

$$
\begin{aligned}
& \sigma_{12}=2\left(c_{44}^{(0)}-\tau_{2} H\left(\varepsilon_{0}\right)\right) \varepsilon_{12}, \\
& \sigma_{13}=2\left(c_{44}^{(0)}-\tau_{2} H\left(\varepsilon_{0}\right)\right) \varepsilon_{13}, \\
& \sigma_{23}=2 c_{44}^{(0)} \varepsilon_{23},
\end{aligned}
$$

involving the quantity

$$
\tau_{2}=c_{44}^{(0)} \frac{2 c_{44}^{(0)} s_{S}}{1+2 c_{44}^{(0)} s_{S}}
$$

Because of the Heaviside function in (11), the relation between shear stress and shear strain becomes nonlinear, too.

When dealing with nonlinear acoustic waves, it is more convenient to work with the first Piola-Kirchhoff stress tensor $\left(T_{\alpha \beta}\right)$ instead of the Cauchy stress tensor $\left(\sigma_{\alpha \beta}\right)$. When transforming from the latter to the prior, additional nonlinear terms appear, which are partly contained in the nonlinear contributions to $\left(T_{\alpha \beta}\right)$ resulting from the matrix material alone. We shall neglect these terms in comparison to the dominant nonlinearity arising from the non-analytic parts (4), (5) and (11). For the purpose of applying perturbation theory, we decompose the first Piola-Kirchhoff stress tensor into the contribution of the matrix material and a perturbation due to the presence of the micro-cracks, $T_{\alpha \beta}=T_{\alpha \beta}^{(0)}+\Delta T_{\alpha \beta}$ with $T_{\alpha \beta}^{(0)}=C_{\alpha \beta \mu \nu}^{(0)} \varepsilon_{\mu \nu}$. The diagonal elements of $\Delta T_{\alpha \beta}$ are given by (4) and (5), when $\sigma$ is replaced by $T$,

$$
\begin{gathered}
\Delta T_{11}=\tau_{0} \varepsilon_{0}+\tau_{1}\left|\varepsilon_{0}\right|, \\
\Delta T_{22}=\Delta T_{33}=\frac{c_{12}^{(0)}}{c_{11}^{(0)}} \Delta T_{11} .
\end{gathered}
$$

For the off-diagonal elements, we find $\Delta T_{23}=\Delta T_{32}=0$ and

$$
\begin{gathered}
\Delta T_{12}=\Delta T_{21}=-2 \tau_{2} \varepsilon_{12}, \\
\Delta T_{13}=\Delta T_{31}=-2 \tau_{2} \varepsilon_{13},
\end{gathered}
$$

if friction is neglected, and

$$
\begin{gathered}
\Delta T_{12}=\Delta T_{21}=-2 \tau_{2} H\left(\varepsilon_{0}\right) \varepsilon_{12}, \\
\Delta T_{13}=\Delta T_{31}=-2 \tau_{2} H\left(\varepsilon_{0}\right) \varepsilon_{13}
\end{gathered}
$$

in the limit of infinite friction coefficient $\bar{\mu}$.

After having established the stress-strain relation for a medium containing micro-cracks that are fully aligned, we comment on the case of a distribution of micro-cracks with totally random orientations. Now, elastic isotropy of the elastic medium is maintained, and the density of potential energy of the medium, considered as a function of macroscopic strain, depends on the three invariants $I_{1}=\eta_{\alpha \alpha}$, $I_{2}=\eta_{\alpha \beta} \eta_{\alpha \beta}, \quad I_{3}=\eta_{\alpha \beta} \eta_{\beta \gamma} \eta_{\gamma \alpha}$ of the Green-Lagrange finite strain tensor $\left(\eta_{\alpha \beta}\right)=\left(u_{\alpha, \beta}+u_{\beta, \alpha}+u_{\gamma, \alpha} u_{\gamma, \beta}\right) / 2$, only. Lyakhovsky and Myasnikov [19] introduced an expression for the density of potential energy that contains a nonanalytic term via its square-root dependence on the strain invariant $I_{2}$,

$$
\Phi=\frac{1}{2} \lambda I_{1}^{2}+\mu I_{2}+\kappa_{L M} I_{1} \sqrt{I_{2}}
$$

We use this potential as a model for an elastic medium containing defects like micro-cracks with perfectly random orientation. In (17), $\lambda$ and $\mu$ are the second-order Lamé constants of the medium with defects, and $\kappa_{L M}$ is an additional constant which becomes zero in the limit of vanishing defect concentration. This potential gives rise to the following dependence of the first Piola-Kirchhoff stress tensor on displacement gradients: $T_{\alpha \beta}=T_{\alpha \beta}^{(0)}+\Delta T_{\alpha \beta}$ with $T_{\alpha \beta}^{(0)}$ being the linear part of $T_{\alpha \beta}$ and

$$
\Delta T_{\alpha \beta}=\left(\delta_{\alpha \lambda}+u_{\alpha, \lambda}\right)\left[\mu u_{\gamma, \lambda} u_{\gamma, \beta}+\kappa_{L M}\left(\sqrt{I_{2}} \delta_{\lambda \beta}+\frac{I_{1}}{\sqrt{I_{2}}} \eta_{\lambda \beta}\right)\right] .
$$


We approximate $\Delta T_{\alpha \beta}$ by keeping on the right-hand side of (18) only the dominant non-analytic contributions,

$$
\Delta T_{\alpha \beta} \approx \kappa_{L M}\left(\sqrt{\varepsilon_{\gamma \lambda} \varepsilon_{\lambda \gamma}} \delta_{\alpha \beta}+\frac{\varepsilon_{\zeta \zeta}}{\sqrt{\varepsilon_{\gamma \lambda} \varepsilon_{\lambda \gamma}}} \varepsilon_{\alpha \beta}\right) .
$$

\section{Rayleigh waves}

For the investigation of the influence of nonlinearity on the propagation of surface acoustic waves, one may follow well-established methods that were pioneered in this context by the authors of [21-25]. The displacement field $\left(u_{\alpha}\right)$, depending on the material coordinates $x_{\alpha}, \alpha=1,2,3$, is written as an asymptotic expansion

$$
u_{\alpha}=v u_{\alpha}^{(I)}+v^{2} u_{\alpha}^{(I I)}+O\left(v^{3}\right),
$$

where the expansion parameter $v$ is a typical strain, and a stretched coordinate $X=v x_{1}$ is introduced.

The first-order field is a linear superposition of a fundamental surface acoustic wave with phase velocity $v_{R}$, frequency $\omega=k v_{R}$ and its higher harmonics,

$$
u_{\alpha}^{(I)}\left(x_{1}, x_{3}, t\right)=\sum_{\ell=1}^{\infty} w_{\alpha}\left(\ell k x_{3}\right) \exp \left(i \ell k\left(x_{1}-v_{R} t\right)\right) A_{\ell}+\text { c.c. }
$$

Here, c.c. stands for the complex conjugate of the preceding expression. We assume that the elastic medium fills the half-space $x_{3}>0$. Consequently, $w_{\alpha}(z)$ decays exponentially for $z \rightarrow \infty$. For homogeneous elastic media without defects, a nonlinear evolution equation for the displacement amplitudes $A_{\ell}$ (with appropriate normalization) was derived [21-25], which may be written in the form

$$
\begin{gathered}
\frac{\partial}{\partial X} A_{\ell}=k^{2} \sum_{m=1}^{\ell-1} m(\ell-m) F(m / \ell) A_{m} A_{\ell-m}- \\
-2 k^{2} \sum_{m=\ell+1}^{\infty}(\ell / m)^{n} m(m-\ell) F^{*}(\ell / m) A_{m} A_{m-\ell}^{*}
\end{gathered}
$$

with exponent $n=1$. The kernel function $F$ in this evolution equation depends on the ratio of two wavenumbers. Its value for the argument $1 / 2$ is directly related to the acoustic nonlinearity parameter (ANP) for surface acoustic waves propagating in the elastic medium in the absence of defects. We call this quantity $\beta_{R}$. For isotropic media, following the definition of the ANP for Rayleigh waves introduced by Herrmann et al. [10,11] and normalizing the functions $w_{\alpha}(z)$ such that

$$
w_{3}(0)=-i \sqrt{1-\left(\frac{v_{R}}{v_{L}}\right)^{2}} \frac{v_{R}^{2}}{2 v_{T}^{2}-v_{R}^{2}},
$$

this relation is

$$
\beta_{R}=8\left(\frac{v_{L}}{v_{R}}\right)^{2} F(1 / 2)
$$

In (23), (24), the quantities $v_{L}, v_{T}, v_{R}$ are the speeds of longitudinal and transverse bulk waves and of Rayleigh waves, respectively. The evolution equation (22) implies that the growth rate for the second harmonic of a timeharmonic surface acoustic input wave is proportional to the square of the input wave's amplitude.

For isotropic media, explicit analytic expressions for the kernel (and hence for the ANP) in terms of the two decay constants of the Rayleigh waves and the two secondorder and three third-order elastic constants are given by Parker [24], Zabolotskaya [25] and Knight et al. [26]. For an isotropic material with density of potential energy

$$
\Phi=\frac{1}{2} \lambda I_{1}^{2}+\mu I_{2}+\frac{1}{6} v_{1} I_{1}^{3}+v_{2} I_{1} I_{2}+\frac{4}{3} v_{3} I_{3}+O\left(\eta^{4}\right),
$$

where $\lambda, \mu, v_{1}, v_{2}, v_{3}$ are the two second-order and three third-order Lamé constants, the ANP has the form

$$
\beta_{R}=b_{0}+b_{1}\left(v_{1} / \mu\right)+b_{2}\left(v_{2} / \mu\right)+b_{3}\left(v_{3} / \mu\right) .
$$

For a given normalization, the coefficients $b_{n}, n=0, \ldots, 3$, depend on the Poisson ratio only.

We now consider the influence of micro-cracks on the nonlinear properties of Rayleigh waves. Treating the stress deviations $\Delta T_{\alpha \beta}$, introduced in (13)-(16), as perturbations, we regard the coefficients $\tau_{n}, n=0,1,2$, as being of first order in the expansion parameter $v, \tau_{0}=v \hat{\tau}_{0}, \tau_{1}=v \hat{\tau}_{1}$, $\tau_{2}=v \hat{\tau}_{2}$. We note that these coefficients can be made arbitrarily small by reducing the concentration of defects.

For simplicity, we consider here only the case of negligible friction within the micro-cracks. In this case, the offdiagonal elements of $\Delta T_{\alpha \beta}$ give rise to a correction of the second-order elastic constants, which is of first order in $v$ and leads to anisotropy, but does not directly affect harmonic generation or nonlinear combination processes to leading order in $v$. Therefore, we shall not account for these off-diagonal components in the following.

When inserting the expansion (20), with (21) for the firstorder field, into the equation of motion for the displacement field and keeping only terms of second order in $v$, we obtain

$$
\begin{gathered}
\rho \frac{\partial^{2}}{\partial t^{2}} u_{\alpha}^{(I I)}-\frac{\partial}{\partial x_{\beta}} C_{\alpha \beta \gamma \delta}^{(0)} \frac{\partial}{\partial x_{\delta}} u_{\gamma}^{(I I)}= \\
=\left(C_{\alpha 1 \gamma \beta}^{(0)} \frac{\partial}{\partial x_{\beta}}+\frac{\partial}{\partial x_{\beta}} C_{\alpha \beta \gamma 1}^{(0)}\right) \times \\
\times \sum_{\ell=1}^{\infty} w_{\gamma}\left(\ell k x_{3}\right) \exp \left(i \ell k\left(x_{1}-v_{R} t\right)\right) \frac{\partial}{\partial X} A_{\ell}+\frac{\partial}{\partial x_{\beta}} \Delta T_{\alpha \beta}^{(I)} .
\end{gathered}
$$

The quantity $\Delta T_{\alpha \beta}^{(I)}$ is obtained by inserting into (13)-(16) the displacement gradients $u_{\alpha, \beta}^{(I)}$ for $\varepsilon_{\alpha \beta}$ and dividing by $v$.

The right-hand side of (27) is a $2 \pi$-periodic function of $\xi=k\left(x_{1}-v_{R} t\right)$. In particular, $\Delta T_{\alpha \beta}^{(I)}$ may be represented as a Fourier series 


$$
\Delta T_{\alpha \beta}^{(I)}\left(x_{1}, x_{3}, t\right)=\sum_{\ell=-\infty}^{\infty} \Delta \tilde{T}_{\alpha \beta}^{(\ell)}\left(x_{3}\right) \exp (i \ell \xi) .
$$

The same argument was used by Pecorari [17] in connection with non-collinear mixing of acoustic bulk waves. A physically meaningful solution for $u^{(I I)}$ has to be a bounded $2 \pi$-periodic function of $\xi$, too, which may be written in the form of a Fourier series,

$$
u_{\alpha}^{(I I)}\left(x_{1}, x_{3}, t\right)=\sum_{\ell=-\infty}^{\infty} U_{\alpha}^{(\ell)}\left(x_{3}\right) \exp (i \ell \xi) .
$$

When inserting (28) and (29) in (27), multiplying both sides of this equation by $w_{\alpha}^{*}\left(n k x_{3}\right) \exp (-i n \xi)$, where $n$ is a positive integer, summing over $\alpha$, integrating over $\xi$ from 0 to $2 \pi$, over $x_{3}$ from 0 to infinity, performing two integrations by parts, thereby obeying the traction-free boundary conditions at the surface, the following infinite set of differential equations is obtained:

$$
i N_{R} \frac{\partial}{\partial X} A_{n}=\int_{0}^{\infty}\left[D_{\beta}(n k) w_{\alpha}\left(n k x_{3}\right)\right]^{*} \Delta \tilde{T}_{\alpha \beta}^{(n)}\left(x_{3}\right) d x_{3} .
$$

Here we have introduced the linear operator $D_{\alpha}(q)=\delta_{\alpha 1} i q+\delta_{\alpha 3} d / d x_{3}$ and the coefficient

$$
N_{R}=2 \rho v_{R}^{2} \int_{0}^{\infty} w_{\alpha}^{*}(z) w_{\alpha}(z) d z .
$$

We note that the quantity $N_{R} / c_{11}^{(0)}$ depends on the Poisson ratio of the matrix material only.

In order to determine the initial growth rate of the $n$th harmonic amplitude for an initially sinusoidal Rayleigh wave, we have to insert the first-order solution (21) with $A_{\ell}=0$ for $\ell \neq 1$ into (13) and evaluate the right-hand side of (30). Defining $\psi$ as the phase angle of $A_{1}$, we obtain

$$
\begin{gathered}
\Delta T_{11}^{(I)}=\left|A_{1}\right|\left[-\hat{\tau}_{0} \sin (\xi+\psi) f_{R}\left(k x_{3}\right)+\right. \\
\left.+\hat{\tau}_{1}\left|\sin (\xi+\psi) \| f_{R}\left(k x_{3}\right)\right|\right],
\end{gathered}
$$

where we have defined the depth profile $w_{\alpha}$ of the Rayleigh waves such that $w_{1}(0)$ is real, and we have defined the real function

$$
f_{R}(z)=2\left[w_{1}(z)-i \frac{c_{12}^{(0)}}{c_{11}^{(0)}} \frac{d}{d z} w_{3}(z)\right] .
$$

With (33) and (34), we obtain from (30)

$$
\frac{\partial}{\partial X} A_{\ell}=k \gamma_{\ell}\left|A_{1}\right|
$$

with the coefficients $\gamma_{\ell}$ being equal to zero for $\ell>1$ odd and

$$
\gamma_{\ell}=\frac{\ell \hat{\tau}_{1}}{(\ell-1)(\ell+1) \pi N_{R}} \mathrm{e}^{i \ell \psi} \int_{0}^{\infty} f_{R}(\ell z)\left|f_{R}(z)\right| d z
$$

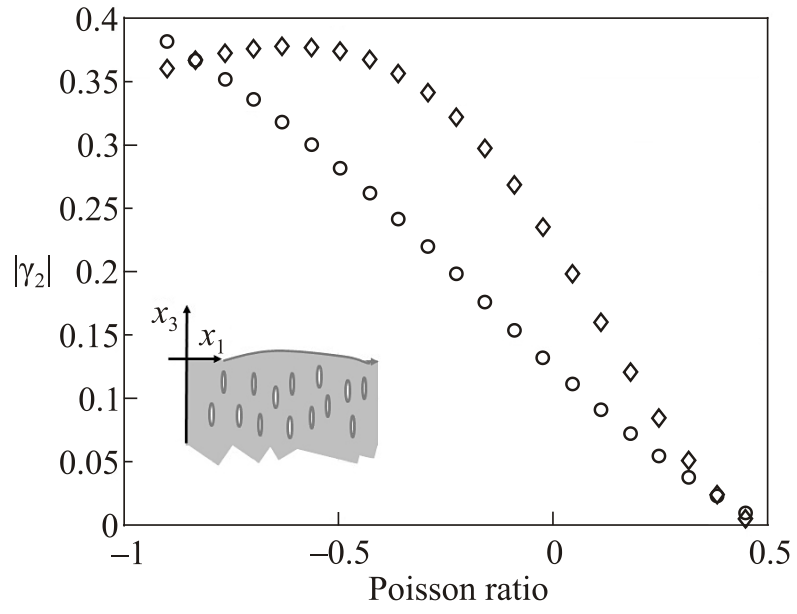

Fig. 1. The quantity $\left|\gamma_{2}\right|$ in units of $0.1 \tau_{1} / \rho v_{R}^{2}$ for fully aligned micro-cracks (diamonds) and in units of $\kappa_{L M} / \rho v_{R}^{2}$ for random orientation (circles) as function of the Poisson ratio. Inset: Orientation of aligned micro-cracks with respect to surface and propagation direction of Rayleigh waves.

for even $\ell$. Note that the modulus of $\gamma_{\ell}$ is independent of the normalization of the depth profile of the Rayleigh wave displacement field. Also, the quantity $\gamma_{\ell} \rho v_{R}^{2} / \hat{\tau}_{1}$ depends on the Poisson ratio of the matrix material, only. This dependence is shown in Fig. 1 for $\ell=2$.

From (34) it also follows that a time-harmonic Rayleigh input wave with amplitude $A_{1}$ generates immediately all even harmonics with initial growth rates being proportional to $\left|A_{1}\right|$. We also note that the initial efficiency of higher harmonic generation decreases, $\gamma_{\ell} \propto \ell^{-2}$ for $\ell \rightarrow \infty$, because the integral on the right-hand side of (35) becomes proportional to $\ell^{-1}$ for large $\ell$.

So far, we have considered Rayleigh waves propagating in a medium with micro-cracks fully aligned according to the inset of Fig. 1. We now move to elastic media containing a distribution of micro-cracks with perfectly random orientation. When adopting the Lyakhovsky-Myasnikov potential (17) as a model for this case, we may proceed in the same way as in the aligned case, using (19) instead of (13)-(16) for the stress deviation $\Delta T_{\alpha \beta}^{(I)}$ and insert there the displacement gradients $u_{\alpha, \beta}^{(I)}$ corresponding to a time-harmonic Rayleigh input wave. It is then straightforward to show that the initial growth of higher harmonics is again described by (34) with coefficients $\gamma_{\ell}$ which can be non-zero only for even harmonics. The dependence of $\gamma_{2} \rho v_{R}^{2} / \kappa_{L M}$ on the Poisson ratio of the matrix material is shown in Fig. 1.

\section{Acoustic wedge waves}

In this section, elastic wedges with opening angle $\theta$ are considered that contain a spatially homogeneous distribution of micro-cracks which are aligned such that the normal of their faces are along the $x_{1}$ direction. The $x_{3}$ direction points along the apex line of the wedge and is the propagation direction of acoustic wedge waves. The 
geometry with the orientation of the coordinate system is shown in Fig. 2. The matrix material is assumed to be isotropic.

In isotropic elastic wedges, acoustic modes may be characterized as either even or odd, depending on their behavior with respect to the reflection at the wedge's midplane $\left(x_{1}=x_{2}\right)$. For opening angles smaller than $90^{\circ}$ and Poisson ratios in the range of most materials of practical use, only odd wedge waves (anti-symmetric flexural modes, ASF modes) exist [27]. Unlike Rayleigh waves, ASF modes do not resonantly generate even harmonics [28]. This means in particular, that the growth rate of the second harmonic of an ASF mode and hence the acoustic nonlinearity parameter of such modes is zero.

For wedge waves of even symmetry, an evolution equation for their slowly varying amplitudes $A_{\ell}$ was derived by Krylov and Parker [29] which can be brought into the form (22) with exponent $n=2$. In analogy to the case of Rayleigh waves, the growth rate of the second harmonic and consequently an acoustic nonlinearity parameter for even acoustic wedge waves would be proportional to the value of the kernel function $F$ in the evolution Eq. (22) at the argument $1 / 2$.

In the following, we shall confine our discussion to ASF modes. The displacement field of a linear time-harmonic wedge wave with wavenumber $k$ and amplitude $A$ has the form

$$
u_{\alpha}\left(x_{1}, x_{2}, x_{3}, t\right)=w_{\alpha}\left(k x_{1}, k x_{2}\right) \exp \left(i k\left(x_{3}-v_{W} t\right)\right) A+\text { c.c. },
$$

where $v_{W}$ is the phase velocity of the wedge wave. The profile functions $w_{\alpha}(x, y)$ have to be normalized appropriately and decay to zero for large distances from the apex line, $\sqrt{x^{2}+y^{2}} \rightarrow \infty$. They may be determined numerically via an expansion in a double series of Laguerre functions after a conformal mapping of the wedge with opening angle $\theta$ into a rectangular wedge [30]. This method was also applied to compute the kernel function $F$ in the evolution Eq. (22) for certain propagation geometries in anisotropic wedges [31].

The distribution of micro-crack orientations shown in Fig. 2 breaks the reflection symmetry with respect to the wedge's midplane, and resonant generation of even harmonics will be allowed. Following the same procedure as
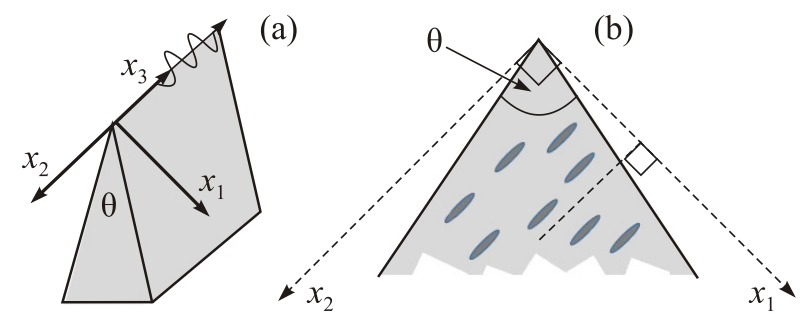

Fig. 2. Wedge geometry. described in the previous section in the context of Rayleigh waves, starting with an asymptotic expansion (20), where the first-order field is now a superposition of wedge waves,

$$
\begin{gathered}
u_{\alpha}^{(I)}\left(x_{1}, x_{2}, x_{3}, t\right)= \\
=\sum_{\ell=1}^{\infty} w_{\alpha}\left(\ell k x_{1}, \ell k x_{2}\right) \exp \left(i \ell k\left(x_{3}-v_{W} t\right)\right) A_{\ell}+\text { c.c. },
\end{gathered}
$$

and the stretched coordinate is $X=v x_{3}$. At second order in the expansion parameter $v$, we obtain an equation of the form (27) with the right-hand side modified. In particular, $w_{\alpha}\left(\ell k x_{3}\right) \exp \left(i \ell k\left(x_{1}-v_{R} t\right)\right)$ is replaced by $w_{\alpha}\left(\ell k x_{1}, \ell k x_{2}\right) \times$ $\times \exp \left(i \ell k\left(x_{3}-v_{W} t\right)\right)$ and $\Delta T_{\alpha \beta}^{(I)}$ depends on all three Cartesian coordinates. In analogy to the case of Rayleigh waves, its dependence on $x_{3}$ is via $\zeta=k\left(x_{3}-v_{W} t\right)$, and it is a $2 \pi$-periodic function of this quantity and may therefore be expanded in a Fourier series of the form (28) with Fourier coefficients $\Delta \tilde{T}_{\alpha \beta}^{(\ell)}\left(x_{1}, x_{2}\right)$. Proceeding now in the same way as in the previous section (see also [31] for the application of this approach), we obtain the analog of (30),

$$
\begin{gathered}
i N_{W} \frac{\partial}{\partial X} A_{\ell}= \\
=\ell k \iint_{S}\left[D_{\beta}(\ell k) w_{\alpha}\left(\ell k x_{1}, \ell k x_{2}\right)\right]^{*} \Delta \tilde{T}_{\alpha \beta}^{(\ell)}\left(x_{1}, x_{2}\right) d x_{1} d x_{2}
\end{gathered}
$$

with

$$
N_{W}=2 \rho v_{W}^{2}(\ell k)^{2} \iint_{S} w_{\alpha}^{*}\left(\ell k x_{1}, \ell k x_{2}\right) w_{\alpha}\left(\ell k x_{1}, \ell k x_{2}\right) d x_{1} d x_{2} \text {. }
$$

In contrast to its definition in the previous section, the linear operator $D_{\alpha}(q)$ in (38) is defined as $D_{\alpha}(q)=\delta_{\alpha 1} \partial / \partial x_{1}+$ $+\delta_{\alpha 2} \partial / \partial x_{2}+\delta_{\alpha 3} i q$. The integrals in (38) and (39) have to be carried out over the cross section $S$ of the wedge in the $x_{1} x_{2}$ plane. Note that $N_{W}$ is independent of $k$ and $\ell$.

We now determine the Fourier coefficients $\Delta \tilde{T}_{\alpha \beta}^{(\ell)}$ for the stress deviation $\Delta T_{\alpha \beta}$ given by the expressions (13)-(15), which correspond to a distribution of aligned micro-cracks as shown in Fig. 2. When inserting in these expressions the first-order displacement field (37) with $A_{\ell}=0$ for $\ell \geq 2$ and $A_{1}=\left|A_{1}\right| \exp (i \psi)$, (38) takes on the form (34). The coefficients $\gamma_{\ell}$ are zero for $\ell$ being an odd integer number. For even $\ell$ they are related to the displacement field of the ASF wedge modes via

$$
\gamma_{\ell}=\frac{i \ell^{2} \hat{\tau}_{1}}{(\ell-1)(\ell+1) \pi N_{W}} \mathrm{e}^{i \ell \psi} \iint_{S} f_{W}(\ell x, \ell y)\left|f_{W}(x, y)\right| d x d y
$$

where we have defined

$$
f_{W}(x, y)=2\left\{\frac{\partial}{\partial x} w_{1}(x, y)+\frac{c_{12}^{(0)}}{c_{11}^{(0)}}\left[\frac{\partial}{\partial y} w_{2}(x, y)+i w_{3}(x, y)\right]\right\} \text {. }
$$

Making use of the isotropy of the matrix material, we define the profile functions $w_{\alpha}(x, y)$ such that $w_{1}$ and $w_{2}$ are 


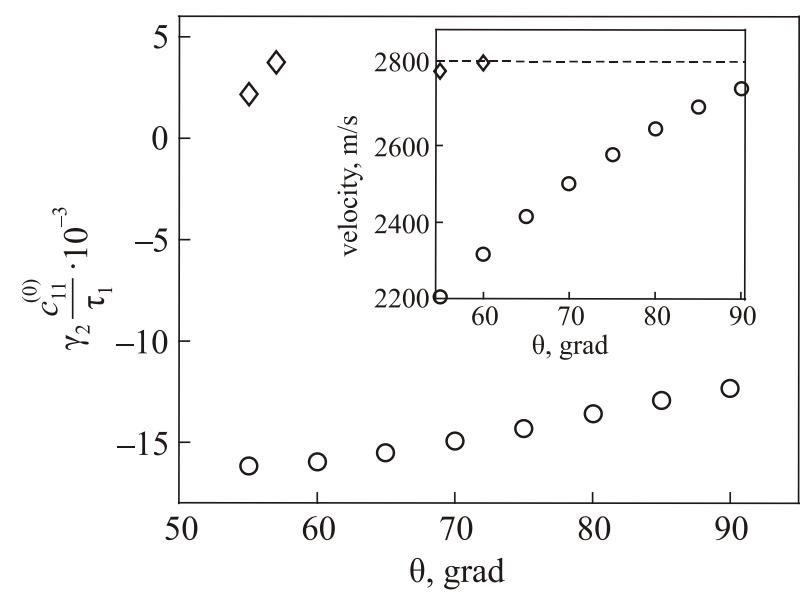

Fig. 3. The dimensionless quantity $\gamma_{2} \cdot c_{11}^{(0)} / \tau_{1}$ as function of the wedge opening angle $\theta$ for steel as matrix material. Inset: Phase velocity of anti-symmetric flexural wedge waves in a steel wedge. Dashed line: Rayleigh wave speed.

real, while $w_{3}$ is imaginary, and consequently $f_{W}$ is real. In the same way as in the case of Rayleigh waves, we find that $\gamma_{\ell}$ is independent of the normalization of the profile functions and that $\gamma_{\ell} \propto \ell^{-2}$ for large $\ell$. In Fig. 3, numerical results are presented for the dependence of $\gamma_{2}$ on the opening angle $\theta$ in the range $55^{\circ} \leq \theta \leq 90^{\circ}$. The quantity $\gamma_{2}$ is the initial growth rate of the second harmonic of a timeperiodic wedge wave apart from the factor $k\left|A_{1}\right|$. The data refer to wedges made of steel with micro-cracks aligned according to Fig. 2(b). The phase angle $\psi$ was chosen to be $-\pi / 4$. The inset of Fig. 3 shows the phase velocity $v_{W}$ of the wedge waves as function of $\theta$. For opening angles $\theta \leq 60^{\circ}$, a second branch of ASF modes exists. The results in Fig. 3 demonstrate that the ASF modes of the branch with higher speed have $\gamma_{2}$ values that differ from those of the branch with lower speed by their sign and have considerably smaller magnitude than the latter.

\section{Conclusions}

In summary, a method has been presented for the calculation of the initial growth rates of higher harmonics of time-harmonic Rayleigh and wedge waves propagating in elastic media with a spatially homogeneous distribution of sub-wavelength micro-cracks. It is based on the effective stress-strain relation of the system, which is non-analytic in the case of flat micro-cracks. Numerical results have been presented for the initial growth rate of the second harmonic.

A basic difference between Rayleigh and anti-symmetric flexural wedge waves pertains to the nonlinearity of the pure matrix material. In the case of Rayleigh waves, the matrix material contributes to the growth rate of the second harmonic a term proportional to the square of the fundamental amplitude, which becomes dominant with decreasing concentration of micro-cracks. In the case of wedge waves, the growth rate of the second harmonic vanishes in the absence of micro-cracks. Consequently, acoustic wedge waves are expected to be particularly sensitive to textured micro-crack distributions that break the reflection symmetry with respect to the wedge's midplane. Since such distributions cause the linear elastic properties of the wedge to become anisotropic, too, there will be a small contribution to the initial growth rate of the second harmonic proportional to the square of the fundamental amplitude, which decreases to zero in the limit of vanishing micro-crack concentration.

A remarkable feature, generated by the non-analyticity of the stress-strain relation and already known for acoustic bulk waves (see, for example, [17]), is the immediate growth of infinitely many (even) harmonics with growth rates depending linearly on the input wave's amplitude. The observability of harmonics higher than the second is clearly limited by attenuation in the metallic samples with defects.

In the case of aligned micro-cracks, the initial growth rates are proportional to the parameter $\tau_{1}$ in the nonanalytic stress-strain relation. This parameter can be quantified by finite-element simulations, which have been carried out for homogeneous distributions of aligned flat micro-cracks in the high-strength metallic alloy IN718 [13]. For the ratio of $\left|\tau_{1}\right|$ and the elastic constant $c_{11}$ of IN718, a value of more than 0.005 was found for a micro-crack concentration of 0.014 per diameter cubed.

Finally we note that the approach presented for the quantitative determination of growth rates of higher harmonics can easily be extended to nonlinear frequency mixing with two guided input waves.

The authors dedicate this work to the memory of Arnold Markovich Kosevich with deep admiration of the valuable contributions he made to the fields of crystal defects and nonlinear waves, which this paper pertains to. One of us (A.P.M.) had the pleasure of cooperating with him on the latter subject.

Financial support by Deutsche Forschungsgemeinschaft (Grant No. MA 1074/11) is gratefully acknowledged.

1. Lord Rayleigh, Proc. London Math. Soc. 17, 4 (1885).

2. P. Lagasse, Electron. Lett. 8, 372 (1972).

3. A.A. Maradudin, R.F. Wallis, D.L. Mills, and R.L. Ballard, Phys. Rev. B 6, 1106 (1972).

4. P. Hess, A.M. Lomonosov, and A.P. Mayer, Ultrasonics 54, 39 (2014).

5. A.P. Mayer, V.V. Krylov, and A.M. Lomonosov, in: Proc. 2011 IEEE International Ultrasonics Symposium (2012), p. 2046.

6. G. Leibfried and W. Ludwig, Z. Phys. 160, 80 (1960).

7. A.M. Lomonosov, P.D. Pupyrev, P. Hess, and A.P. Mayer, Phys. Rev. B 92, 014112 (2015).

8. V.E. Nazarov and A.M. Sutin, J. Acoust. Soc. Am. 102, 3349 (1997).

9. I. Solodov, D. Döring, and G. Busse, Strojniški Vestnik Journal of Mechanical Engineering 57, 169 (2011). 
10. J. Herrmann, J.Y. Kim, L.J. Jacobs, J. Qu, J.W. Littles, and M.F. Savage, J. Appl. Phys. 99, 124913 (2006).

11. K.H. Matlak, J.Y. Kim, L.J. Jacobs, and J. Qu, J. Nondestruct. Eval. 34, 273 (2015).

12. A. Korobov, M. Izossimova, A. Kokshaiskii, and A. Agafonov, in: AIP Conf. Proc. 1685, 080005 (2015).

13. M. Rjelka, B. Koehler, and A.P. Mayer, unpublished.

14. M. Kachanov, Appl. Mech. Rev. 45, 304 (1992).

15. Y. Zhao, Y. Qiu, L. Jacobs, and J. Qu, Acta Mechanica 227, 399 (2016).

16. Y. Zhao, Y. Qiu, L. J. Jacobs, and J. Qu, J. Appl. Mech. 82, 081006 (2015).

17. C. Pecorari, Wave Motion 59, 69 (2015).

18. T. Oberhardt, J.-Y. Kim, J. Qu, and L.J. Jacobs, AIP Conf. Proc. 1706, 020024 (2016).

19. V.A. Lyakhovsky and V. Myasnikov, Fizika Zemli 10, 71 (1984) [Phys. Solid Earth 10, 72 (1984)].

20. V.P. Smyshlyaev and J.R. Willis, J. Mech. Phys. Solids 44, 49 (1996).
21. V.P. Reutov, Izv. Vuzov. Radiofizika 16, 1690 (1973) [Radiophys. Quant. Electron. 16, 1307 (1973)].

22. N. Kalyanasundaram, R. Ravindran, and P. Prasad, J. Acoust. Soc. Am. 72, 488 (1982).

23. R.W. Lardner, Int. J. Eng. Sci. 21, 1331 (1983).

24. D.F. Parker, Int. J. Eng. Sci. 26, 59 (1988).

25. E.A. Zabolotskaya, J. Acoust. Soc. Am. 91, 2569 (1992).

26. E.Yu. Knight, M.F. Hamilton, Yu.A. Il'insky, and E.A. Zabolotskaya, J. Acoust. Soc. Am. 102, 1402 (1997).

27. G.L. Zavorokhin and A.I. Nazarov, Zapiski Nauchnykh Seminarov POMI 380, 45 (2010) [J. Math. Sci. 175, 646 (2011).]

28. V.V. Krylov and D.F. Parker, Wave Motion 15, 185 (1992).

29. V.V. Krylov and D.F. Parker, unpublished.

30. S.L. Moss, A.A. Maradudin, and S.L. Cunningham, Phys. Rev. B 8, 2999 (1973).

31. E.S. Sokolova, A.S. Kovalev, and A.P. Mayer, Wave Motion 50, 246 (2013). 